\title{
ASEGURAMIENTO DE LA CALIDAD EDUCATIVA EN MÉXICO. ALGUNAS CONSIDERACIONES FUNDAMENTALES
}

\section{ASSURANCE OF EDUCATIONAL QUALITY IN MEXICO. SOME FUNDAMENTAL CONSIDERATIONS}

\section{Joaquín Aurelio Lezama Valdés.}

Asesor Académico.

Universidad Pedagógica Nacional, Unidad 211, Puebla, México.

Correo: joaquin_401@hotmail.com

\section{RESUMEN}

En este artículo se reflexiona, a partir de una revisión documental, sobre aquellos aspectos que en un primer momento fueron relegados de los procesos de evaluación y acreditación de la calidad educativa, pero que actualmente están develando su relevancia para el aseguramiento de la misma y convirtiéndose en ejes sustantivos para el mejoramiento genuino de las instituciones de educación superior. Se enfatiza la importancia de los docentes para el logro de calidad y se determinan conceptos fundamentales para una nueva aproximación al aseguramiento de la calidad educativa.

\section{PALABRAS CLAVE}

Acreditación, calidad de la educación, docencia, evaluación, ética. / Accreditation, education quality, teaching, evaluation, ethics.

\section{ABSTRACT}

In the present article we reflect, on the basis of a documental review, on the aspects that in a first moment were neglected from processes of evaluation and accreditation of education quality, but that have been proving their relevance for its assurance and becoming substantial axes for the true improvement of higher education institutions. It is emphasized the importance of 
teachers for the achievement of quality and it is determined core concepts for a new approach to education quality assurance.

\section{INTRODUCCIÓN}

Las decisiones de las últimas décadas del gobierno mexicano en cuanto a educación han sido la arena conceptual en donde como es natural que suceda, algunas ideas dominantes se han vuelto hegemónicas al verse traducidas en políticas que guían el quehacer de las instituciones educativas, incluidas las universidades. Los términos utilizados en textos oficiales e institucionales dan cuenta de ello. Con el último cambio de régimen, se han modificado los discursos del Estado dando un giro importante a las prioridades en la nación. Escuchamos más ahora sobre combate a la corrupción y la recuperación de la dignidad de la profesión docente que acerca de gobernabilidad o competitividad.

Este cambio de enfoques en el país pudiese parecer caprichoso e infundado si no estamos bien informados de los acontecimientos globales y la preocupación internacional por subsanar las carencias que la evaluación educativa y la acreditación ha revelado a partir de los resultados reales, limitados, obtenidos por las IES tras la implantación de modelos rígidos, impositivos y técnicos. Por ejemplo, Lemaitre (2017), en un documento publicado por la UNESCO sobre América Latina, deja en claro que hay una demanda actual urgente por el desarrollo de una segunda generación de mecanismos de aseguramiento de la calidad cuya pertinencia y eficacia se fundamente en la capacidad de atender las complejas necesidades de las universidades.

En éste artículo se hace una revisión documental, reflexiva, sobre la relación entre algunas de las nuevas consideraciones y los procesos de evaluación y acreditación de instituciones de educación superior que desea enfocarse en los efectos colaterales y omisiones que han deteriorado los intentos de mejora en las IES.

\section{ACREDITACIÓN Y CALIDAD EDUCATIVA}

Salas (2013: 310) define a la acreditación de la calidad educativa como una herramienta que permite "sistematizar los procesos con la doble finalidad de, por una parte, buscar la eficiencia en el manejo de los recursos $y$, por otra, contar con elementos para tomar mejores decisiones". Esto, aclara el autor, difiere de la creencia errónea de que la acreditación es una "fórmula operativa para cuantificar la calidad educativa". El mismo autor agrega, con respecto a lo educativo, que se debe considerar a la acreditación como un medio que "permita generar información confiable acerca de los procesos académicos que derive en la toma de mejores decisiones, lo cual es distinto de tratar de reducir la calidad educativa a indicadores, objetivos y metas" (p. 328). Otra definición de calidad en éste ámbito es la de Buendía (2011: 6) expresada como "el grado de ajuste entre las acciones que una institución, programa académico o carrera pone en marcha para dar cumplimiento a las orientaciones emanadas desde su misión y propósitos institucionales y los resultados que de estas acciones obtiene".

La acreditación de la calidad educativa, según Stensaker (2013) tiene varios propósitos entre los cuales están el establecer estatus, legitimidad o adecuación, hacer juicios sobre desempeño, auditar, autorizar, otorgar licencias y reconocimientos formales estatales a programas de estudio, individuos o grupos de individuos, instituciones y organizaciones. Este trabajo, dice el autor, comúnmente implica garantizar la rendición de cuentas, verificar resultados de aprendizaje en la práctica, crear canales de información dirigidos a estudiantes y empleadores, evaluar instituciones nuevas (enseñanza, personal, infraestructura y currículo), apoyar la delegación de poder del estado a instituciones autónomas, y, ayudar a la 
movilidad estudiantil proporcionando información sobre títulos y comparativos internacionales de las IES.

\section{ACREDITACIÓN Y CORRUPCIÓN}

Lamentablemente, las agencias acreditadoras son susceptibles de participar en acciones de corrupción, definida esta última por Waite y Allen (2003: 282) como "cualquier uso de poder o posición a través de actos discretos o comportamiento (s) que benefician a un individuo, grupo, u organización". Estas conductas obscuras e inapropiadas se generan en los procesos de acreditación, afirma Guidó (2005), porque cuando una universidad solicita una acreditación, la intención con lo que lo hace puede inclinarse más a ser una legitimación que una búsqueda honesta de superación. El mismo Guidó resalta que esto acontece de forma diferente en IES públicas y privadas. En las últimas, comenta el investigador, se tiende a ver a la acreditación como un factor de mercadeo competitivo y atracción de clientes potenciales. En el caso de las universidades estatales, acreditar proporciona los medios para rendir cuentas sobre el uso de recursos asignados y para obtener nuevos financiamientos. En ambos casos se generan presiones que fomentan actos de corrupción. Aunque, no debemos descartar la posibilidad de que, como aclara Aguilera (2017), aún si se tiene una lógica empresarial de gestión orientada al lucro (legítimo, por supuesto), las IES pueden ver en la acreditación una oportunidad de cambiar sus horizontes. Esta perspectiva no será romántica ni altruista sino práctica, a condición de darse cuenta de que, para la captación de matrícula, además del marketing, existen factores como la demanda creciente de calidad en servicios educativos.

La corrupción en la evaluación para la acreditación ha sido confirmada por diferentes investigaciones. Buendía (2011) destaca la simulación (indicadores que no se cumplen realmente ni favorecen reflexión crítica); las relaciones clientelares y mercantilizadas de universidad-mercado laboral y universidadorganización acreditadora, y, la burocratización que afecta a la gestión institucional. Salas (2013) encontró que las IES pueden simplemente buscar definir, sistematizar, medir y evaluar procesos para que nada cambie. Es decir, hacer lo mismo, pero con la diferencia de que se almacenan evidencias de ejecución. Martínez, Tobón y Romero (2017) explican, acerca de factores que lesionan los procesos de evaluación, que los modelos de acreditación tienden a enfocarse más en lo administrativo que en desempeños; que se simula (contratando investigadores de renombre sólo por prestigio y eliminando datos negativos, por ejemplo), que, se desarticulan los sectores administrativo y académico. Además, dicen ellos, se centraliza el control de la acreditación a una persona o grupo reducido pues no se busca la participación de la comunidad. Finalmente, mencionan la ausencia de credibilidad en los organismos acreditadores, generada por procesos complejos, oscuros, descontextualizados y limitados al llenado de formatos. Esto puede hacer, como explican los últimos autores mencionados, que acreditación no sea sinónimo de aseguramiento de la calidad.

Hay factores, explica Cárdenas (2012), que fomentan la corrupción tales como el monopolio (oferta limitada contra gran demanda de educación o control de plazas), discrecionalidad (autoridades que no monitorean o carecen de facultades para sancionar, regulación poco clara y excesiva, impunidad, coaliciones políticas, y falta de estándares), y, deficiente rendición de cuentas (falta de monitoreo administrativo, colusiones entre autoridades, ausencia de transparencia y de escucha a los principales beneficiarios del servicio: alumnos y padres de familia). Están también las reglas poco claras para los maestros, falta de monitoreo y guía por parte de colegas, ambiente de impunidad, percepción de la enseñanza como una profesión temporal, con bajos salarios y compensaciones 
sociales. Adicionalmente está el proceso de hechura de políticas centralizado que descuida y hasta asegura la falta de voz de los maestros. Agrega el investigador que una condición necesaria para lograr equidad y calidad en la educación es la reducción en la frecuencia de los actos de corrupción. Con ello concuerdan Waite y Allen (2003), quienes afirman que los sistemas y los individuos corruptos victimizan a las personas, atrayéndolas hacia la participación en conductas incorrectas que tenderán a apuntalarse consumiendo tiempo, dinero y energía, pues las acciones realizadas dentro de un sistema corrompido se volverán a la vez formas y productos de la corrupción. Como consecuencia, llevar a cabo un proceso de acreditación en condiciones de corrupción responderá sólo a intereses particulares.

Cárdenas (2012) asegura que directores, docentesysupervisorespuedengenerarprácticas de corrupción como soborno, clientelismo, incumplimiento de responsabilidades, nepotismo, cobro de comisiones o cuotas ilegales o fantasmas, ausentismo y cleptocracia. Tras lo comentado podremos ver mejor que el combate a la corrupción educativa no es un asunto de moda sino una condición necesaria sin la cual el logro de calidad quedará siempre debilitado y cuestionado. Una paradoja es que las instituciones más débiles, o sea, las que necesitan más imperiosamente del acompañamiento de procesos de acreditación para crecer y mejorar son las que, confirma Heyneman (2013) ceden más fácilmente a las presiones colocadas sobre ellas por la competencia de recursos, fama y notoriedad, pues carecen todavía de medios de control o gestión consolidados.

Heyneman (2013) aporta a las prácticas de corrupción en las universidades arriba mencionadas el soborno pagado por aspirantes a ingresar a programas o IES específicas, fraude financiero a través de empresas fantasma
- gastos ficticios, fábricas de títulos (que ofrecen títulos en corto tiempo y bajo costo), empresas de acreditación falsas, programas internacionales (que favorecen el surgimiento de agentes que cobran comisión e instituciones de dudosa reputación), exageración del éxito de los egresados en el mercado laboral (para atraer clientes) y falta de regulación en la integridad de los estudiantes.

Como se puede ver, combatir la corrupción en la educación no es una tarea fácil por la variedad de formas en que se presenta. Sin embargo, debe ser una de las prioridades de las naciones, aseguran Hallak y Poisson (2005), quienes distinguen tres campos respecto de la corrupción en la educación. Primeramente, el poder monopolizado y la falta de mecanismos de rendición de cuentas que favorecen el desarrollo de prácticas corruptas. Luego, los comportamientos de los actores que tienen un impacto significativo sobre el acceso, calidad y equidad en la educación. Finalmente, el hecho de facilitar el acceso a la información y promover una "voz de los ciudadanos" es una acción que resulta esencial para mejorar tanto la transparencia como la rendición de cuentas en el uso de recursos. Además, los autores describen algunos momentos, en los que la corrupción surge en las instituciones educativas y en los que habría que poner especial atención: cuando se construye o rehabilita una escuela, adquiere equipos, libros o comida, se designan puestos o dirige a profesores y sus conductas, se aplican examinaciones y otorgan diplomas, manipulan o seleccionan medios de información, entregan bonos específicos y manejan finanzas. Ante esto, en una publicación posterior, Poisson (2010) advierte que la utilización de medidas aisladas es insuficiente para reducir el fraude y la corrupción en la educación. Ella aconseja una acción concertada de un "triángulo virtuoso" que abarca estos frentes principales: el desarrollo de sistemas transparentes de regulación y establecimiento de estándares, 
construcción y fortalecimiento de la capacidad de control (management) y mayor apropiación de la comunidad del control de los procesos administrativos y financieros.

Esta triada, concuerda con lo expresado por Transparencia Internacional y por el Concejo Internacional de Política de Derechos Humanos (2010), agrupaciones que aseveran que la participación, transparencia y acceso a la información, además de ser elementos efectivos para la lucha anti-corrupción, pueden fortalecer los derechos humanos en procesos que aspiran a proveer acceso y calidad en servicios, entre los cuáles se encuentra la educación. Lo que es más, ellos aseguran que implementar el enfoque de derechos humanos fortalece las medidas anti-corrupción que también deben ocuparse de la discriminación de individuos y grupos vulnerables, pobreza y cuestiones de género. Existen, según explica Transparencia Internacional, estándares de provisión de servicios basados en leyes internacionales, mismos que incluyen lo siguiente: a) disponibilidad (suficiencia en cantidad y calidad para satisfacer necesidades de la comunidad en cuestión), b) accesibilidad (que los servicios se asignen y proporcionen a la comunidad sin discriminación y estén al alcance de forma física y económica pero además, aseguren acceso a la información), c) aceptabilidad (se deben respetar los valores locales en cuanto a forma y contenido de acuerdo a la cultura de la comunidad en cuestión); y, d) adaptabilidad (Ios servicios deben adaptarse a las necesidades de comunidades $o$ individuos en diferentes contextos sociales y culturales).

Hallak y Poisson (2007) formulan las siguientes recomendaciones a los países que quieren combatir la corrupción educativa, en donde según sus estimados, las malas prácticas pueden representar hasta un $80 \%$ del presupuesto no salarial asignado a educación. Dicen los expertos que se debe asegurar el cumplimiento de dos requisitos previos. En primer lugar, hay que revelar qué tipos de corrupción y qué vínculos existen entre ellos en cada contexto en particular. Y, también, tomar medidas simultáneas en diferentes áreas: política, legal, institucional y gerencial, económicas y educativas. Una vez hecho lo anterior, se podrá trabajar en los siguientes factores internos: a) establecer claramente procesos y responsabilidades; b) conservar registros de pagos y compras y tener procesos de auditoría regularmente; c) ejercer una supervisión efectiva que asegure el cumplimiento de reglas: d) detectar señales de riesgo, $y, e)$ proveer canales adecuados para preguntas y quejas, así como la divulgación de un sistema de penalización para quien no se adhiera a lo establecido. Existen factores externos que requerirán atención como de la formación de líderes (sindicales y universitarios, por ejemplo), favorecimiento de la competencia para eliminar monopolios, reforzar el derecho a la información por medio de la transparencia y rendición de cuentas, acción colectiva de todos los implicados y un enfoque integral que tome en cuenta la educación en la ética.

Salas hace 5 recomendaciones para facilitar la adopción de un proceso de acreditación con resultados exitosos que, pensamos, seguidas con rigor, además de proporcionar un plan de acción claro, pueden coadyuvar para evitar la implantación de procesos de acreditación desvirtuados. Estas sugerencias son: a) Capacitación de la organización acerca de los conceptos asociados a la calidad educativa; b) Adaptación de la estructura y organigrama junto a la revisión de funciones; c) Combate de la resistencia al cambio por medio de líderes académicos convincentes; d) Replanteamiento de las funciones docentes de acuerdo criterios actualizados; $y, e)$ sistemas robustos de gestión de la información (Salas, 2013: 312-314)

\section{ENFOQUES DE LA ACREDITACIÓN}

El enfoque desde el cual una institución 
decide adoptar un modelo de acreditación condiciona su consolidación en la realidad. A continuación, se discutirá la conveniencia de un cambio de perspectiva para la obtención de logros educativos tangibles que no siempre son el resultado de elegir el camino más corto y cómodo. Etxague, Huegun, Lareki, Sola y Aramendi (2009) distinguen tres paradigmas de evaluación y acreditación dominantes: tecnológico, interpretativo y sociocrítico. El primero utiliza referentes externos de tipo empresarial para buscar alcanzar resultados prefijados. El segundo concibe a las instituciones como universos construidos por los propios actores desde adentro hacia afuera sobre marcos teóricos. El Tercero critica los modelos impositivos cuestionando sus ejes (mercantilismo, eficiencia y eficacia), que impulsan un lenguaje con términos como cliente, liderazgo y proveedores, y una ideología subyacente de individualismo, competitividad extrema, relativismo moral, falta de equidad, elitismo, olvido de los desfavorecidos e imperio de las leyes del mercado. Estos autores aclaran que el modelo más técnico es el que ha logrado imponerse en las gestiones de calidad de las instituciones educativas. A partir de lo dicho, se puede deducir que, el modelo tecnológico, al ser externo, se ve como una imposición que se tiene que cumplir, sin necesidad de dedicar esfuerzos para construcciones conceptuales ni involucramiento consentido de los participantes o consideraciones sociales, culturales, humanistas o éticas. Si bien esta linealidad unidireccional permitirá ahorrar tiempo y energía a las universidades, también contiene el alto costo de disminuir los efectos benéficos de los procesos de acreditación.

A ese respecto, Gómez y Marecos (2017) aseveran que para consolidar procesos de calidad se deberá ir más allá de las políticas de regulación y contar con sistemas internos de autorregulación que verdaderamente induzcan progresos hacia la conquista de estándares apropiados. Un obstáculo que surge de las universidades, comentan los expertos, es defender y utilizar una perspectiva meramente funcional (como es la tecnológica), que impedirá apreciar la dinámica de la vida institucional, pues, al buscar solamente dar cumplimiento a las exigencias de un ente acreditador no se propiciará una genuina cultura de la autoevaluación, dando paso a visiones parciales. Esto hace necesario contar con la mirada crítica e integral de los directamente involucrados para aspirar al fortalecimiento e innovación institucional. Especialmente será una piedra angular la participación comprometida de los profesores, pues no puede existir calidad académica sin confianza y sin prácticas apropiadas en el aula.

\section{RESPETO A LA AUTONOMÍA $Y$ CONFIANZA EN LAS ACREDITADORAS}

Para Gómez y Marecos (2017), la confianza en la relación entre agencias acreditadoras y universidades es axiológica. Esto demanda la inclusión de las opiniones de todas las personas involucradas en procesos de acreditación para construir propósitos, objetivos y metas que las instituciones deben cumplir. Es indispensable, aseveran los expertos, que se genere un circuito virtuoso, en el que tanto la auto-evaluación como la evaluación externa produzcan aprendizajes y propuestas fundamentadas de innovación. Esto es, superar lo meramente técnico para dar cabida a los conocimientos tácitos, saberes lingüísticos, arte y tradición transmitidos a las generaciones en formación por medio del diseño y utilización de indicadores que den cuenta de la totalidad de los participantes de los procesos educativos. La condición fundamental para tal confianza será el respeto a la autonomía, pues para transformar vidas y realidades, se requiere de libertad académica e intelectual. La censura y los intereses políticos, económicos o sectarios, evitará que se genere bien común. La comunidad debe entender a la ES como 
eso, un bien común, y fomentar el pensamiento crítico hacia el interior de las instituciones como punto de partida de propuestas pedagógicas. En el texto de Gómez y Marecos se advierte claramente que los procesos de evaluación realizados por las acreditadoras son incapaces por sí mismos de aumentar la calidad. Ello sucederá sólo a partir de la voluntad de mejora de las instituciones. Los estímulos, orientaciones, presiones y penalizaciones de las acreditaras serán poco útiles sin la colaboración comprometida de las universidades.

\section{REPENSAR EL ROL E INFLUENCIA DEL DOCENTE PARA LA MEJORA DE LAS IES.}

Si nos preguntamos por los atributos comunes a las instituciones educativa exitosas, Namo (2005) enlista lo siguiente: "liderazgo pedagógico, autonomía, visión y metas compartidas, centrada en contenidos básicos, ambiente favorable para el aprendizaje, altas expectativas, seguimiento del progreso del alumno, evaluación institucional, refuerzo positivo, capacitación centrada en la práctica, facilitación de las instancias centrales del sistema, y, colaboración con la familia" (P. 29). Es necesario preguntarse si existe la posibilidad de lograr verdadera calidad en los aspectos mencionados sin contar con el trabajo eficaz y comprometido del profesorado. Por ello nunca se podrá enfatizar suficientemente el amplio valor de sus contribuciones.

Actualmente, el papel protagónico del docente se está volviendo una certeza, reporta Robalino (2005: Pp. 8-9), quien lo expresa de ésta manera: "iSin docentes, los cambios educativos no son posibles!". Por esto mismo resulta preocupante constatar que a los profesores, dice la especialista, "se les exige hacer todo lo que la sociedad, los estados y la familia no están haciendo". Y, que las exigencias relacionadas a "la calidad, eficacia y competitividad no siempre van acompañadas de recursos, autonomía y cambios estructurales". Es decir, existe una falta de corresponsabilidad y acompañamiento al docente que puede deberse, según explica Robalino, a la percepción tradicional que se tiene de éste como un ejecutante de currículos diseñados externamente; visión que descarta su contribución a los aprendizajes de los estudiantes, la gestión institucional y las políticas educativas. No es de extrañarse entonces que los docentes se sientan agobiados, acotamos concordando con Frenzel y Rojas (2007), por la imposición de normas externas que definen lo "justo y razonable", el "deber ser" muchas veces cristalizado por una cultura política que no les facilita su labor.

Noda (2017), establece que la cohesión entre los diferentes actores de una institución es fundamental para evitar esa paradoja en la que por un lado se anuncia que el recurso humano es "el activo más valioso" y por el otro, por medio de las políticas, actitudes y valores ejercidos en la práctica, se le menosprecia, generando desconfianza e incredulidad, desmotivación y falta de compromiso. Esto sucede, puntualiza la autora, cuando hay inconsistencia entre lo que se piensa, escribe, dice y se hace al interior de las instituciones. Por eso, el logro de calidad requiere de líderes institucionales capaces de atraer, retener, mantener, motivar y desarrollar personal idóneo y comprometido que ayude a alcanzar los objetivos institucionales emanados de su misión y visión mediante la colaboración de la comunidad en los procesos de diseño, ejecución e implementación.

Re-significar el rol del docente no será simple pues implicará más que una simple petición de opinión. Habrá que reconsiderar "la formación inicial, el desarrollo profesional en servicio, condiciones de trabajo, salud, autoestima, compromiso profesional, clima institucional, valoración social, capital cultural, salarios, estímulos, incentivos, carrera profesional y evaluación del desempeño" (Robalino, 2005p.12). En resumen, es lógico pensar que el compromiso esperado en los maestros se 
pueda generar mejor como respuesta recíproca a la corresponsabilidad adquirida por las IES y las acreditadoras que por políticas impositivas que niegan a los docentes la facultad de incidir directamente sobre los procesos educativos para luego responsabilizarlos de aquello que pudiese ir mal.

La búsqueda de la armonía entre los actores internos y externos de la acreditación, vista como corresponsabilidad, contradice la lógica neoliberal de la estandarización en la que, según comenta Fardella (2012), se prescribe y determina por medio de descriptores externos la manera en la que los docentes deben conducirse. El desacato de los profesores a esos descriptores, o sea, actuar fuera del sistema, provocará que los docentes se vean invisibilizados y excluidos. En ese sentido los perfiles profesionales descontextualizados se vuelven factores de inclusión y exclusión que dejan sin posibilidad de interpelación al maestro, forzándolo a inscribir su práctica profesional dentro de un lenguaje ajeno, de racionalidad eficientista. Por ello, las acreditadoras, al estar conscientes de que su trabajo debe servir como apoyo externo a los logros que cada IES se propone alcanzar contando con la contribución armónica de sus docentes, evitará convertirse en instrumento de fuerzas de poder homogeneizantes que se valen de palabras sutiles para construir verdades absolutas que silencian las voces importantísimas de actores fundamentales de la educación.

Así llegaremos a la conclusión de que, como en un proceso de colaboración no debe haber absolutismos, en lugar de importar conceptos de calidad que pueden no corresponder a la realidad de las universidades, se le debe otorgar a los docentes, así como a los demás miembros de la comunidad educativa, oportunidad de construir un concepto de calidad pertinente para la consecución de los objetivos institucionales.

\section{EVALUACIÓN DOCENTE}

Ya establecida la importancia de los docentes para la calidad educativa, podremos entender por qué su evaluación es vital. Las instituciones pueden recurrir para ello a listados de criterios sobre las responsabilidades y conductas de profesores. Estas enumeraciones se encuentran en publicaciones de agrupaciones expertas. Un ejemplo de ellas es la Organización Internacional del Trabajo (OIT en español o ILO por sus siglas en inglés), misma que acerca del perfil del docente calificado determina: tiene conocimiento de su disciplina, habilidades pedagógicas, aprendizaje dinámico, capacidad para evaluar, cuidar del bienestar de los alumnos a su cargo y ser auto-formativo. A esto se agrega el trabajo colaborativo tanto en el aula como con la institución, poder atender la diversidad en el aprendizaje, generar cohesión con la institución, hacer planeación, poder tomar decisiones profesionales, establecer relaciones de guía con los padres y lograr enlaces con comunidades externas. A lo anterior se suman las recomendaciones que la organización toma de la UNESCO para ámbitos universitarios: satisfacer las necesidades de enseñanza para todos los alumnos incluyendo el desarrollo de pensamiento crítico y el intercambio de ideas, realizar investigación que influya tanto al interior de las universidades como hacia comunidades externas y contribuir a la gobernabilidad de la institución participando en trabajo colegiado (OIT, 2012).

En el mismo texto de la OIT se establecen criterios y principios de la evaluación docente, misma que debe ser: formativa, ligada a las estrategias, sobre evaluación y objetivos de la institución, sostenida por criterios holísticos, transparente, objetiva, equitativa y justa, balanceada en cuanto a las necesidades de mejora y retroalimentación y, basada en autoregulación profesional. 
Aunque, como ya ha sido mencionado, la evaluación para la acreditación debe superar las limitaciones de las definiciones técnicas e incluir espectros más amplios para poder reflejar las realidades institucionales con mayor precisión. Por ejemplo, los acreditadores deben ir más allá de la simple verificación de los nombres escritos en publicaciones y estar conscientes que la exigencia por publicar genera conductas no éticas. Entre ellas se encuentran la permisividad por parte de los docentes hacia el trabajo de los alumnos presentado con irregularidades, plagio, condicionamiento y presión de los centros de trabajo hacia sus maestros para conseguir $o$ mantener el empleo, falseamiento de información y apropiación de crédito por quien no realizó la investigación, por mencionar algunos aspectos (Hirsch, 2016). Heyneman (2013) confirma que las presiones sobre los profesores en entornos de competencia y mercantilización pueden llevarlos a un comportamiento corrupto, sobre todo si la comunidad no proporciona recompensas suficientes ni equitativas al buen comportamiento, incluidos los profesores adjuntos, y, si se privilegia la investigación sobre la enseñanza como criterio de excelencia.

Una propuesta más se puede deducir del estudio de Etxague, Huegun, Lareki, Sola y Aramendi (2009). Quienes solicitaron la opinión de profesores para conocer el impacto de los procesos de acreditación en su lugar de trabajo. Ellos tomaron en cuenta variables como las aportaciones de entrada o inputs de la institución (recursos, apoyos innovación y formación docente), los procesos educativos (con subcategorías como lo curricular, lo organizativo y atención a la diversidad), los resultados (en imagen del centro, humano y epistemológico en los alumnos y de objetivos académicos). A partir de su investigación se pudo constatar la utilidad de las percepciones de los profesores para conocer los aspectos en los que un proceso de acreditación ha obtenido beneficios, así como en donde no se han alcanzado los objetivos propuestos. La relación que esto tiene con la evaluación docente es que el contacto de evaluadores y docentes, contextualizado, puede representar una valiosa oportunidad para convertir sus interacciones, normalmente unidireccionales dentro de un modelo técnico de evaluación, en diálogos capaces de aportar información importante para el bien común.

Esa unidireccionalidad vertical es lo que pudo verificarse por un estudio sobre 78 universidades públicas mexicanas publicado por Rueda, Luna, García y Loredo (2010). Su investigación reporta aspectos interesantes en cuanto al impacto de la evaluación de docentes: Aunque se detenta la mejora de la docencia como objetivo central no se utilizan los resultados de las evaluaciones para mejorar la enseñanza. Ellos quedan dentro del ámbito administrativo traduciéndose en estímulos al desempeño, promoción, permanencia y aumentos salariales, evaluaciones de acreditación externa o programas especiales del gobierno. En el caso de estímulos, sólo se considera a los profesores de tiempo completo, dejando fuera a los de tiempo parcial. En la mayoría de las instituciones, el cuestionario de apreciación estudiantil es el principal instrumento utilizado. El hecho de que la mayoría $(77 \%)$ de las universidades diseñan sus propios cuestionarios, genera dudas sobre la correspondencia entre las preguntas y las necesidades de la institución o que se tenga intención de resolver los problemas detectados por medio del instrumento. Esta falta de certeza crece cuando se constata que las capacitaciones de las instituciones generalmente no tienen conexión con los hallazgos de las evaluaciones. Además, los investigadores comentan que los participantes indicaron que los responsables de realizar las evaluaciones no se encuentran bien capacitados en la materia y suelen tener escolaridad y profesiones muy diversas. Algo notorio es que a los docentes se les da retroalimentación, pero no se promueven oportunidades para discutir los hallazgos en 
grupos o en cuerpos académicos o de áreas. Con ello también se pierden oportunidades de mejora institucional, pues, como explican Zabalza, Cid y Trillo (2014), el desafío es orientar el cambio no a los individuos sino al contexto institucional. Ellos comentan que, si un profesor introduce cambios en sus lecciones, aunque interesante, resulta de poco impacto. Serán aquellas modificaciones que comprometan tanto a varias instancias institucionales como las que afectan al proyecto educativo de las instituciones los que incrementan la influencia de la innovación. En otras palabras, la formación docente orientada a cambios grupales debería ser una prioridad en las universidades.

\section{CÓDIGOS DE ÉTICA POR Y PARA LOS DOCENTES}

Si las universidades y agencias acreditadoras renunciasen a la relación vertical con sus profesores, de poder y control, para permitir interacciones colaborativas, horizontales, descubrirían las bondades que tratar a los docentes como profesionales expertos pueden traer al bienestar de todos. Una herramienta que el profesorado puede diseñar y aplicar, tiene incluso el potencial para sustituir en gran medida los reglamentos y normatividad impositivos. Este instrumento es el código de ética. La razón para emplear códigos de ética para el impulso de la calidad docente la explica extraordinariamente Van Nulan:

"El buen maestro conoce y aplica una variedad de estrategias de enseñanza al presentar contenidos, trata a los estudiantes con respeto, reflexiona acerca de cómo mejorar la enseñanza, $y$ completa la gran cantidad de actividades asociadas con la enseñanza y la escuelas. Esta descripción de un maestro habla del fin técnico de la enseñanza, pero no es el núcleo de la enseñanza. La integridad está en el núcleo de ser un maestro: al final del día, la integridad (como un aspecto de la ética y la conducta) es lo que permanece" (2009: 88).

Los códigos de ética no son una receta mágica infalible. Sin embargo, pueden convertirse en poderosas herramientas para la lucha contra la corrupción y generar y mantener orden consensuado en las universidades. Poissson (2009; 2010) comenta que los códigos de ética para docentes contribuyen de dos maneras. Primero, apoyan en la construcción de mejores entornos e interacciones de enseñanza y aprendizaje mientras ayudan a frenar conductas no deseadas. Segundo, al promover la ética, pueden asegurarse de que los valores comunes sean compartidos por todos los implicados. La autora explica que éstos listados detallan las obligaciones de los maestros tanto en su enseñanza (competencia y asistencia regular) como en sus relaciones con la administración, colegas, alumnos y la comunidad en general (imparcialidad, no discriminación). Ella agrega que para que su aplicación se debe tomar en cuenta la participación de los profesionales de la docencia y sindicatos de docentes en el desarrollo en su implementación, dar fácil acceso y difusión (en formación docente y para profesores en servicio), establecer mecanismos necesarios para el registro y trámite de cualquier queja a nivel nacional, local o escolar; y promover la autorregulación por parte de la comunidad de docentes. Poisson establece los siguientes pasos para iniciar el diseño y uso de códigos de ética en una institución: formular el contenido del código, redactarlo, diseminarlo y promoverlo, implementarlo, reportar y sancionar conductas inadecuadas y revisar y evaluar su impacto.

\section{PAPEL DE LOS EVALUADORES}

Aguilera (2017), acertadamente establece el reto que representa formar evaluadores para procesos de acreditación, pues, dado que el máximo conocimiento se encuentra en las universidades, entonces, ¿quién estará 
capacitado para evaluarlas? Y, ¿qué criterios pueden utilizarse sino aquéllos que las mismas instituciones provean? Estas dos cuestiones dan forma a los sistemas de evaluación y acreditación de las IES. Son, los académicos más consolidados quienes evalúen la calidad, pero, para evitar que el proceso sea endógeno, mayormente se ha decidido que un instituto (generalmente una agencia) organice y coordine las actividades de evaluación y acreditación. Los evaluadores, por provenir del mundo académico son pares de los evaluados. Ellos tendrán que establecer principios que generalmente se deducen del ser y quehacer de las universidades. Para ello habrá que reflexionar sobre el ser y sentido de las IES en estos tiempos sin dejar de lado su origen y desarrollo histórico. Aguilera también acepta que los estándares de calidad deben construirse desde las mismas IES, por lo que el rol de las acreditadoras será el de coordinar y establecer pautas metodológicas de trabajo, es decir, referentes tanto académicos como de gestión para las universidades.

\section{CONCLUSIONES.}

Resumiendo lo argumentado a lo largo de éste artículo, los cambios provocados por los tiempos actuales exigen a las universidades una transformación genuina, que abandone la simulación y que tenga claridad en un aspecto central: cualquier intento que se haga de lograr calidad apoyándose en el acompañamiento de la evaluación tiene tendencia a desdibujarse fácilmente debido a la multiplicidad de intereses e interacciones en juego. Por eso, el combate a la corrupción de la educación y de su evaluación debe convertirse en una prioridad. Se deberá revalorar la integridad, honestidad y actitudes éticas no desde una postura idealista sino como único camino hacia la verdadera evolución de la educación. Asimismo, se tendrá que reconocer el papel protagónico del docente dentro de las universidades y dignificar su trabajo como colaborador profesional cuya aportación es un componente insustituible para la calidad educativa resulta imperativo.

Es también sustancial repensar la evaluación docente como un acompañamiento de carácter horizontal en el que todos los involucrados comparten la responsabilidad por establecer las condiciones para que el profesorado lleve a cabo su labor formadora sin ser coaccionado por presiones alienantes de su trabajo o acciones punitivas que no aspiran a la mejora de la enseñanza, sino que persiguen la mercantilización indiscriminada o la legitimación. Para ello, las evaluadoras y acreditadoras deberán superar los criterios técnicos, tan impulsados por la visión funcional neoliberal pero insuficientes para describir, y por lo tanto cambiar, la realidad de las IES. Este cambio puede generarse por ejes como la transparencia, la participación y el acceso a la información, para poder con ello conseguir una reducción de los altos costos, económicos y sociales, que la corrupción genera en el sistema donde se sitúa la enseñanza superior.

Además, la confianza entre evaluadores y el respeto a la autonomía de las instituciones y a la libertad intelectual y de cátedra de los docentes se revela como un ingrediente sustancial para la vitalidad y funcionamiento colaborativo de las IES. La confianza en las acreditadoras generada no por la imposición ni por deseos de perpetuación de discursos oficiales sino por procesos claros y transparentes también es esencial para cumplir en su papel de agentes externos de cambio capaces de apoyar con su conocimiento y experiencia a las universidades. Ello con el fin de lograr propuestas de mejora auto-generadas y auto-reguladas confeccionadas desde el interior de las instituciones pero que armonizan con los contextos externos más amplios.

Daremos conclusión a éste texto aclarando que su factura no responde a deseos de legitimación de las políticas del gobierno en turno. Los discursos del pasado sirvieron para expresar 
una serie de objetivos que, estemos de acuerdo o no con ellos, sirvieron de guía a la educación en el país. Pero, el tiempo todo lo cambia y el presente reclama la capacidad de superar lo concluido y elaborar nuevos modelos de evaluación y acreditación, más comprehensivos, con una perspectiva distinta, con la finalidad de que sus alcances sean mayores y logren impactar en la realidad siempre perfectible da las universidades y de la sociedad en general. Subyacentes a los términos destacados existen conceptos complejos que reflejan la cotidianeidad de las IES y de sus posibles aspiraciones por progresar. Es muy probable que seguiremos teniendo encuentros con estas ideas más allá de la presente administración nacional, pues rara vez los discursos oficiales en materia de educación responden a caprichos o improvisaciones de un hombre sólo.

\section{REFERENCIAS BIBLIOGRÁFICAS}

Aguilera, R. (2017) Aseguramiento de la calidad de la educación superior. En: Educación Superior y Sociedad (ESS) Instituto Internacional de Unesco para la Educación Superior en América Latina y el Caribe (IESALC ) Colección 25 aniversario Vol. 22 ISSN 07981228. Pp. 131154.

https://unesdoc.unesco.org/ark:/48223/ pf0000261633

Buendía, A. (2011) Evaluación y acreditación de programas en México. Más allá de los juegos discursivos. En: Revista diálogos sobre educación. Año 2, No 3, julio-diciembre 2011. ISSN 2007-2171. Pp. 1-19

http://dialogossobreeducacion.cucsh.udg.mx/ index.php/DSE/article/view/371

Cárdenas, S. (2012). La corrupción en sistemas educativos: una revisión de prácticas, causas, efectos y recomendaciones. Revista Electrónica de Investigación Educativa, 14(2), 52-72. http://redie.uabc.mx/vol14no2/contenidocardenas.html
Consejo Internacional de Política de Derechos Humanos-Transparencia Internacional (2010) Integrating human rights in the anticorruption agenda. Challenges, possibilities and opportunities. Suiza.

https://assets.publishing.service.gov.uk/ media/57a08b34e5274a27b2000a0f/ integrating-humrights_.pdf

Etxague, X., A. Huegun, A. Lareki, J. Sola y P. Aramendi (2009) Diferencias en la percepción de variables educativas antes y después de la implantación de sistemas de acreditación de calidad. En: Revista iberoamericana sobre calidad, eficacia y cambio en la educación. 2009, Vol 7, No 3. ISSN: 1696-4713.

https://www.redalyc.org/pdf/551/55114063002. pdf

Fardela, C. (2012) Verdades sobre la docencia, efectos y consecuencias subjetivas de la evaluación docente en Chile. EN: Revista de Psicología, Vol. 21, № 1, junio 2012. Pp. 209227.

https://www.researchgate.net/ publication/273028455_Verdades_sobre la docencia efectos y consecuencias subjetivas de la evaluacion docente en $\underline{\text { Chile }}$

Frenzel y Rojas (2007) Impacto de los procesos de evaluación y acreditación en el ámbito universitario (ponencia) Facultad regional tucumán - universidad tecnológica nacional. VII coloquio internacional sobre gestión universitaria en América de Sur. "Movilidad, gobernabilidad e integración regional" Mar del Plata: Argentina. 29 de noviembre a 1ro de diciembre del 2007.

https://repositorio.ufsc.br/bitstream/ handle/123456789/82949/TRABAJOFRENZEL. pdf? sequence $=1 \&$ is Allowed $=y$

Gómez y Marecos (2017). Regulación versus 
mejora continua de la calidad de la educación superior: equilibrio entre la evaluación externa y gestión interna, en el marco de la autonomía institucional. En: Educación Superior y Sociedad (ESS) Instituto Internacional de Unesco para la Educación Superior en América Latina y el Caribe (IESALC Colección 25 aniversario Vol. 22 ISSN 07981228. Pp. 21-41.

http://catedrarsu.udg.edu/admin/js/ kcfinder/upload/files/N\%C3\%BAm\%20 26\%20Revista $\% 20$ Educaci $\%$ C $3 \%$ B 3n $\% 20$ Superior $\% 20 y \% 20$ Sociedad.pdf

Guidó, E. (2005) Acreditación: ¿calidad o instrumento de legitimación para la educación superior? En: Revista Electrónica "Actualidades Investigativas en Educación" Volumen 5, Número Especial, Año 2005, ISSN 1409-4703: Costa Rica.

http://www.cea.ucr.ac.cr/media/diea/ acreditacion.pdf

Hallak, J., \& Poisson, M. (2005). Ethics and corruption in education: an overview. Journal of Education for International Development, 1(1).

http://equip123.net/JEID/articles/1/1-3.pdf

Hallak, J. y Poisson, M. (2007) Corrupt schools, corrupt universities: What can be done? Francia: UNESCO-International Organization for EducationPlanning.

https://unesdoc.unesco.org/ark:/48223/ pf0000150259

Heyneman, S. (2013) Higher education institutions. Why they matter and why corruption puts them at risk. En Sweeney, Despota y Lindner (eds.) Global Corruption Report: Education TRANSPARENCY INTERNATIONAL. P. 101-107.

https://web.edu.hku.hk/f/acadstaff/376/ Global\%20Corruption \%20Report\%3A\%20 Education.pdf
Hirsch, A. (2016) Comportamiento responsable en la investigación y conductas no éticas en universidades de México y España. En: Revista de la Educación Superior 45(179) (2016) 79-93.

http://publicaciones.anuies.mx/pdfs/revista/ Revista179_S2A5ES.pdf

Lemaitre, M. (2017) Presentación. En: Educación Superior y Sociedad (ESS) Instituto Internacional de Unesco para la Educación Superior en América Latina y el Caribe (IESALC Colección 25. aniversario Vol. 22 ISSN 07981228. Pp. $11-20$.

http://catedrarsu.udg.edu/admin/js/ kcfinder/upload/files/N\%C3\%BAm\%20 26\%20Revista\%20Educaci\% C3\%B3n $\% 20$ Superior $\% 20 y \% 20$ Sociedad.pdf

Jorge E. Martínez, J., S. Tobón y A. Romero (2017) Problemáticas relacionadas con la acreditación de la calidad de la educación superior en América Latina. En: Innovación Educativa. ISSN: 1665-2673 vol. 17, número 73 | enero-abril, 2017. Pp. 79-96.

http://www.scielo.org.mx/pdf/ie/v17n73/16652673-ie-17-73-00079.pdf

Namo, G. (2005) Profesores para la igualdad educacional en América Latina. Calidad y nadie de menos. En: Revista PRELAC Proyecto Regional de Educación para América Latina y el Caribe. No 1/Julio de 2005. Protagonismo docente. Pp 25-37.

https://unesdoc.unesco.org/ark:/48223/ pf0000144666

Noda, M. (2017) Evaluación de la calidad y su acreditación en Cuba: actualidad y retos en el contexto de la agenda educativa 2030. En: Educación Superior y Sociedad (ESS) Instituto Internacional de Unesco para la Educación Superior en América Latina y el Caribe (IESALC Colección 25. ${ }^{\circ}$ aniversario Vol. 22 ISSN 07981228. Pp. 87-109. 
http://catedrarsu.udg.edu/admin/js/ kcfinder/upload/files/N\%C3\%BAm\%20 $26 \% 20$ Revista $\% 20$ Educaci $\%$ C $3 \%$ B $3 n \% 20$ Superior $\% 20 \mathrm{y} \% 20$ Sociedad.pdf

Organización Internacional del Trabajo (2012) Handbook of good human resource practices in the teaching profession. International Labour Office: Suiza.

https://www.ilo.org/wcmsp5/groups/public/--ed_dialogue/---sector/documents/publication/ wcms_187793.pdf

Poisson, M. (2009) Guidelines. For the design and effective use of teacher codes of conduct. France: UNESCO-International Institute for Educational Planning.

https://unesdoc.unesco.org/ark:/48223/ pf0000185010

Poisson, M. (2010) Corruption and education. Francia: UNESCO

https://www.iaoed.org/downloads/WEB 11 corruption\%20and\%20education final .pdf

Robalino, M. (2005) ¿Actor o protagonista? Dilemas y responsabilidades sociales de la profesión docente. En: Revista PRELAC Proyecto Regional de Educación para América Latina y el Caribe. No 1/Julio de 2005. Pp. 7-24.

https://unesdoc.unesco.org/ark:/48223/ pf0000144709

Rueda, M, E. Luna, B. García y J. Loredo (2010) La evaluación de la docencia en las universidades públicas mexicanas: un diagnóstico para su comprensión y mejora. En: Revista Iberoamericana de Evaluación Educativa 2010 - Volumen 3, Número 1e. ISSN: 1989-0397. Pp. 77-92.

https://repositorio.uam.es/bitstream/ handle/10486/661585/RIEE_3_1_6. pdf?sequence $=1$
Salas, I. (2013) La acreditación de la calidad educativa y la percepción de su impacto en la gestión académica: el caso de una institución del sector no universitario en México. En: Calidad en la Educación n. o 38, julio 2013 • pp. 305-333

https://scielo.conicyt.cl/scielo.php?script=sci_art text\&pid=S0718-45652013000100009

Stensaker, B. (2013) Ensuring quality in quality assurance. En Sweeney, Despota y Lindner (eds.) Global Corruption Report: Education Transparencia Internacional. P. 124-132.

https://web.edu.hku.hk/f/acadstaff/376/ Global\%20Corruption\%20Report\%3A\%20 Education.pdf

Van Nulan, S. (2009) Teacher codes: learning from experience. Francia: Unesco-International Institute for Educational Planning.

https://unesdoc.unesco.org/ark:/48223/ pf0000185872

Waite, D. y D. Allen (2003) Corruption and Abuse of Power in Educational Administration. En: The Urban Review, Vol. 35, No. 4, December 2003.

https://www.academia.edu/31369277/ Corruption and Abuse of Power in Educational_Administration

Zabalza, M., A. Cid y F. Trillo (2014) Formación docente del profesorado universitario. El difícil tránsito a los enfoques institucionales. En: Revista Española de Pedagogía. Año LXXII, No 257, enero-abril 2014, Pp. 39-54.

https://revistadepedagogia.org/wp-content/ uploads/2014/02/257-03.pdf

(⿸丆口

